\title{
Two Different Minimally Invasive Approaches for Management of Thoracolumbar Fractures
}

Chenghao Yu ( $\square$ yuchenghaosyd@163.com )

The First People's Hospital of Jingmen

\section{Fan Ding}

The First People's Hospital of Jingmen

\section{Xiaosong Wu}

The First People's Hospital of Jingmen

\section{Zhengyun Ye}

The First People's Hospital of Jingmen

\section{Bing $\mathrm{Hu}$}

Tianyou Hospital affiliated to Wuhan University of Science and Technology

\section{Research article}

Keywords: Thoracolumbar Fractures, Percutaneous Pedicle Screw Fixation, Minimally invasive, Wiltse paraspinal approach

Posted Date: November 1st, 2021

DOl: https://doi.org/10.21203/rs.3.rs-970783/v1

License: (c) (i) This work is licensed under a Creative Commons Attribution 4.0 International License. Read Full License 


\section{Abstract}

Objective To compare the clinical effect and safety of pedicle screw fixation via percutaneous approach and Wiltse paraspinal approach for thoracolumbar fractures without neurological deficit.

Methods 98 cases who suffered from single level thoracolumbar fracture without nerve injury were treated by pedicle screws fixation via either percutaneous approach (percutaneous group) and Wiltse paraspinal approach(paraspinal group). Perioperative indexes, imaging parameters and functional and symptom results of the two groups were recorded and compared.

Results All patients were followed for more than 12 months, and the incision length and postoperative hospital stay in the percutaneous group were significantly shorter than those in the paraspinal group $(P<0.05)$, intraoperative blood loss was less than that of the paraspinal group $(P<0.05)$, operative and postoperative costs and the number of fluoroscopy were significantly higher than those of the paraspinal group $(P<0.05)$. There was no significant difference in operative time between the two groups $(P>0.05)$. The anterior edge height percentage of the injured vertebrae and kyphosis Cobb Angle were significantly improved 1 week and 1 year postoperatively in each group $(P<0.05)$, there was no statistical difference between the two groups $(P<0.05)$. As for Visual Analog Scale (VAS) scores, in each group there were continuous decreases 3 days, 6 months, and 1 year postoperatively $(P<0.05)$; There were no statistically significant differences between the two groups before operation, 6 months and 1 year postoperatively $(P<0.05)$, but a significant difference 3 days postoperatively $(P<0.05)$. In terms of Oswestry disability index (ODI), in each group there was continuous decreases 6 months and 1 year postoperatively $(P<0.05)$; and there was no significant difference between the two groups $(P<0.05)$. There was no significant difference in the accuracy of implant between the two groups $(P<0.05)$. In the percutaneous group, there were 2 cases of incision fat liquefaction, 1 case of guidewire fracture and 1 case of the anterior wall of the vertebra penetrated by guide wire rupture. 1 diabetic case of superficial incision infection and 2 cases of skin edge necrosis were found in the paraspinal group.

Conclusion In the treatment of thoracolumbar fractures without neurological defect, pedicle screw fixations via Wiltse paraspinal and percutaneous approach both can obtain minimally invasive and reliable effect, but the percutaneous approach bring smaller trauma, less blood loss, longer operation time, more fluroscopy, higher surgery and postoperative costs, with its own unique complications especially in early learning curve.

\section{Introduction}

Thoracolumbar fractures are the most common type of spinal fractures[1, 2]. In China, the average annual incidence of spinal fractures was 33 per 100,000, in which the thoracolumbar fractures occurred most frequently [3]. Although nonoperative management of thoracolumbar fractures in some neurologically intact patients could obtain favorable clinical outcomes [4], surgical interventions often provide relatively better therapeutic outcomes by stable internal fixation [5]. Posterior pedicle screw 
internal fixation has been considered as the effective method in the treatment of thoracolumbar vertebral fractures because of its advantages of three-dimensional orthodontic and rigid fixation. However, in conventional open surgery, extensive dissection of paravertebral muscle and continuous traction of soft tissue are always associated with local muscle ischemic necrosis, denervation and fibrosis, which may contribute to intractable stiffness of the waist and back, affecting the prognosis of patients $[6,7]$. With the development of imaging and internal fixation techniques, the concept of minimally invasive spinal surgery is gradually accepted by the majority of spinal surgeons. So far minimally invasive spinal surgery for thoracolumbar fractures including pedicle screw fixation by percutaneous approach and Wiltse paraspinal approach has obtained satisfactory outcomes. However, few studies comparing the clinical effect and safety of pedicle screw fixation via the two approaches above for thoracolumbar fractures without neurological deficit have been performed. In this study, we aimed to review 2 years of clinical cases to assess clinical and radiological outcomes of the two approaches for thoracolumbar fractures.

\section{Materials And Methods}

From June 2017 to June 2019, 98 cases who suffered from single level thoracolumbar fracture without nerve injury were treated by pedicle screws fixation via either percutaneous approach and Wiltse paraspinal approach. All patients had preoperative anteroposterior and lateral radiographs, computed tomography (CT) scan, magnetic resonance imaging(MRI)of the thoracolumbar or lumbar spine and bone density measurement. CT scans were taken to classify the fracture type, to evaluate vertebral comminution, and to see whether the pedicles were intact or not, while MRI can demonstrate the occupation of spinal canal and reveal whether spinal cord and nerve root are compressed.

Case selection criteria were as follows: (1) diagnostic criteria were : clear history of spinal trauma; local pain, waist movement disorders, kyphosis deformity, imaging result confirming $A O$ Type- $A 2, A 3$ and $B 1$ single segmental thoracolumbar vertebral body fracture; (2) the spinal canal of the responsible segment is not invaded or the occupation of spinal canal is $\leq 30 \%$, and the pedicle and facet joints are intact; (3) there were no symptoms of spinal cord or nerve root damage, no need to do spinal canal decompression; (4) an age between 18 and 60 years, and the course of disease within 2 weeks. Patients with serious cardiopulmonary insufficiency and other underlying diseases which may not be able to endure the operation, with severe multiple injuries, or with osteoporosis and pathological fracture were excluded.

The general data including gender, age, body mass index, fracture segment, AO spine classification and injury causes were collected and recorded.

\section{Surgical procedure}

Surgical procedures for all enrolled patients were performed by a single spinal surgeon team. The patients were turned prone on a radiolucent surgery table under general anesthesia and radiography can be completed throughout a full range of $360^{\circ}$. Chest and pelvis were supported by gel pads to make the abdomen off. The procedure was performed under the assistance of a C-arm image intensifier. 


\section{Surgical technique of percutaneous group}

The image intensifier was oriented in an accurate anteroposterior direction to locate the fractured vertebra, and the body surface projections of the pedicles of adjacent vertebral bodies were marked with the help of a custom-made metal grid locator. The lumbar area was then prepared and draped in a sterile fashion. A 1-to 2-cm longitudinal incision was made $1 \mathrm{~cm}$ lateral to the projection of the pedicle. A puncture needle was inserted with its tip on the lateral margin of the pedicle oval and advanced until the tip abutted the bone under anteroposterior view. The proper entry point of puncture needle point is 9-10 o 'clock to the left pedicle and 2-3 o 'clock to the right pedicle, and it should be advanced at $10-15^{\circ}$ angle of the sagittal orientation. A small progress was made in the cortex before the image intensifier was adjusted to a lateral view. The lateral view showed the needle passing parallel to the upper endplate. Minor adjustment was sometimes required. When the needle tip arrived at the posterior vertebral wall on lateral view, intrapedicular location of the needle was confirmed to remain lateral to the medial margin of the pedicle on anteroposterior view. A guide wire took the place of the needle core to get a satisfactory penetration and location in the vertebra, and the needle was removed with care to maintain the location of the guide wire, followed by insertion of a 5-stage metallic tubular dilator. The first 4 stage sleeves were taken out, and the 5th stage sleeve was left to protect the surrounding soft tissue. With the guide wire still in place, a hole on the cortical bone at the entry point was drilled using a 5.0-mm cannulated drill bit. After dilation of the channels and tapping with a thread tap, a pedicle screw was screwed into the prepared hole with the same orientation as the guide wire under the fluoroscopic guidance. Similar procedures were repeated on the other target pedicles, followed by insertion of pre-curved titanium rods. The nuts were screwed in, and tighten after satisfactory reduction was achieved by a dedicated distraction device and examined with the $\mathrm{C}$-arm X-ray. The nail tails were broken, after which the incisions were irrigated and closed.

\section{Surgical technique of Wiltse paraspinal group}

A standard posterior midline incision was made based on the location of the injured vertebra. The cautery dissection was carried successively to expose the lumbodorsal fascia. The fascia was cut open $1.5 \mathrm{~cm}$ bilateral to the supraspinous ligament and the spatium between the longissimus and the multifidus muscle was sought and dissected bluntly until to the exterior margin of the zygapophysial joint. Pedicle screws were inserted bilaterally by hand into the vertebraes adjacent to the injured one, and their positions were ensured by anteroposterior and lateral fluoroscopy images.

Two pre-curved rods with proper lengths were fixed, and distraction was done according to the state of reduction. The nuts were locked when the reduction was satisfied. The nail tails were broken, and the wound was closed after the drainage tube was placed.

\section{Evaluation indicators}

The collection, measurement and record for all evaluation indicators of all patients were completed by a single surgeon. The perioperative parameters including the incision length, operative time, blood loss, 
fluoroscopy number, operative and post-operative costs, postoperative hospital stay and complications of patients in the two groups were recorded. Imaging parameters including Cobb angle of kyphosis and percentage of anterior vertebral height from the two groups were evaluated before and 1 week and 1year after surgery. Visual Analog Scale (VAS) of the back pain and Oswestry disability index (ODI) score were examined to evaluate symptoms and function outcomes at preoperative, and 6 months and 1 year after operation. The percentage of anterior vertebral height of injured vertebra $=$ Actual height $/$ Reference height $x 100 \%$. Reference height $=$ the sum of upper and lower anterior vertebral height of injured vertebra/2. Accuracy of screw placement was assessed by postoperative CT scans, and evaluated by the grade system described by Mobbs and Raley [8] (Grade 0: screw within cortex of pedicle; Grade 1: screw thread breach of wall of pedicle $<2 \mathrm{~mm}$; Grade 2: significant breach $>2 \mathrm{~mm}$ with no neurological compromise; Grade 3: complication including pedicle fracture, anterior breach with neuro-vascular compromise, and lateral or medial breach with neurological sequelae).

\section{Statistical analysis}

The software Statistical Package for the Social Sciences (SPSS, version 19.0) was used for all statistical analyses. The measurement data was expressed as mean \pm standard deviation, and performed using Student's $t$ test. The enumeration data was compared by the chi-square test. A p-value $<0.05$ was considered statistically difference.

\section{Results}

The percutaneous group showed better than the paraspinal group in the incision length, intraoperative blood loss and postoperative hospital stay $(P<0.05)$, but it suffered significantly more fluoroscopy time as well as larger operative and post-operative costs $(P<0.05)$. There was no significant difference in operative time between the two groups (Table 2).

Compared with pre-operation, the Cobb angles were statistically decreased and the percentage of anterior vertebral height were statistically increased 1 week postoperative and 1 year postoperative in both groups $(P<0.05)$, but there was no statistical difference between the two groups in the Cobb angle and the percentage of anterior vertebral height before surgery, 1 week postoperative and 1 year postoperative $(P>$ 0.05) (Table 3).

In each group VAS scores 3 days postoperative, 6 months postoperative and 1 year postoperative were statistically lower than pre-operation (all $P<0.05$ ). There was no significant difference in VAS scores between the two groups before surgery and 6 months postoperative $(P>0.05)$, but VAS scores 3 days postoperative in the percutaneous group were statistically lower than that in paraspinal group $(P<0.05)$. No statistically significant difference was detected in VAS scores 1 year postoperative between two groups $(P>0.05)$. There was no significant difference in ODI scores between the two groups before surgery, 6months postoperative and 1 year postoperative $(P>0.05)$ (Table 4). 
According to the grade system described by Mobbs and Raley, Grade 1 was observed in 4/192 pedicle screws in the percutaneous group and 6/200 in the paraspinal group. Grade 2 was observed in 3/192 pedicle screws in the percutaneous group and 4/200 in the paraspinal group. There was no Grade 3 screw misplacement. No statistically significant difference was found between two groups $(P>0.05)$ (Table 5).

Postoperative incision healing was good in percutaneous group except two cases of fat liquefaction exudation, which were healed by dressing change in a local hospital. Other complications in percutaneous group included one case of guide wire rupture and one case of the anterior wall of the vertebra penetrated by guide wire. The broken guide wire was removed by pulling out the screw. The anterior wall of the vertebra was penetrated by guide wire when tapping with a thread tap, but fortunately the guide wire did no damage to the great vessels. One case of superficial infection with a long history of type 2 diabetes and two cases of skin edge necrosis were found in paraspinal group, all of which were healed by dressing change in local hospitals. No other complications were found in paraspinal group. No implant dislodgement, screw loosening or breakage was detected before instrument removal in either group. There was no significant difference in the incidence of complications between the two groups ( $P$ > 0.05).

\section{Discussion}

In 1968, Wiltse first described the paraspinal sacrospinalis-splitting approach [9], and later he replaced the originally designed bilateral incisions with a single median incision and expanded the indications of this approach [10].

Via the anatomical avascular space existing between the multifidus and the longissimus muscles, this procedure provides an access to the intervertebral foramen, the facet joints, and the transverse processes. Although it still belongs to a type of open approach, Wiltse paraspinal approach not only avoids extensive detaching of the paraspinal muscle, electrical-burn damage and continuous retractor compression for neurovascular supply, but also preserve the posterior osseous structure including the zygapophysial joint, and the posterior ligamentous complex to the maximum extent.

By imaging, histology and electrophysiological assessment, Liu et al [11] found that the Wiltse approach for thoracolumbar fractures was accompanied with a lower incidence of multifidus atrophy and denervation, and less fatty infiltration compared with conventional posterior open approach. Pedicle screw fixation via Wiltse paraspinal approach has been considered as an effective and minimally invasive approach in treatment of thoracolumbar fracture with neurologic intact for the advantages of simple operation and less trauma $[12,13]$.

In 1995, Mathews et al. [14] apply percutaneous pedicle targeting originally designed for temporary external fixation to perform an entirely percutaneous lumbar pedicle fixation, and later Foley et al. [15] suggested improved percutaneous techniques. 
Following each incision which was 1.5-2cm long and down to the deep fascia, the insertion of puncture needle and the placement of screws were carried out under the guidance of the fluoroscopy with small damage to paravertebral muscle, which avoids iatrogenic injuries to the posterior ligamentous complex, minimizes surgical exposure, reduces intraoperative blood loss, and has a better postoperative pain score than open surgery, thereby improving the clinical outcome. After measuring cross-sectional areas of the multifidus muscle by the preoperative and postoperative MRI, Kim et al [16] detected significant decrease in open pedicle fixation group but no statistical difference in percutaneous fixation group. An increasing number of studies suggested that percutaneous pedicle screw fixation has the advantages of limited injury, less bleeding, shorter hospital stay, reliable immediate fixation and better pain improvement [1719]. A recent meta-analysis also confirmed those study results [20]. The present study compared the clinical and radiological effects of the two procedures for mono-segmental without neurological injury. Meanwhile, perioperative parameters, operative and post-operative costs and complications related to the two procedures also were compared.

Spinal fusion has always been taken as the role of the stabilising procedure for a long time [21], but some scholars suggested that in traditional open surgery for thoracolumbar fracture no significant differences existed in clinical or radiologic outcome between the fusion group and no fusion group, and fusion procedure was associated with increased operative duration and larger intraoperative bleeding [22]. A recent systematic review and meta-analysis also drew similar conclusions [23]. Furthermore, compared with open short-segment pedicle screw fixation with posterolateral fusion or no fusion, percutaneous pedicle screw placement without fusion could provide not only similar radiologic outcome but also earlier pain relief and functional improvement [18, 24]. Liu et al. [25] also reported that no statistically difference existed between the two groups with no fusion in radiologic outcome and the accuracy rate of screw placement, however, Wiltse paraspinal approach had apparent advantages over the conventional open method in operative time, intraoperative blood loss, postoperative drainage, and postoperative pain improvement. In our study, no fusion was completed in the two groups. We found that in both groups the Cobb angles and the anterior vertebral height were statistically improved 1 week and 1 year after surgery compared with pre-operative data, and there was no statistical difference between the two groups before and after surgery. Pedicle screw fixation after hyperextended position reduction under general anesthesia could correct kyphosis and restore the anterior vertebral height, but loss of correction and kyphotic angle increase is an inevitable problem observed in the follow-up of the surgical treatment for thoracolumbar fractures. The follow-up results indicated that the percutaneous and Wiltse paraspinal approach groups did not show significant differences in short and long-term radiological effect, and that both approaches can bring satisfactory reduction effects.

This is different from the results of Fan et al. [26]. In their study, uniaxial screws were used in both surgical procedures, and the results showed that the correction of the deformity in the paraspinal group was significantly better than that in the percutaneous group. They think that the skin and muscles in the percutaneous procedure may hinder indirect reduction, while the paraspinal approach provides the nail placement and reduction in nearly direct vision. Fitschen-oestern et al. [27] found that percutaneous surgery and open surgery showed similar early results in reduction and reduction loss, and there was no 
significant difference in reduction loss between the two surgeries over time. There was no significant difference between uniaxial and multiaxial screws. In this study, uniaxial screws were also used in both groups. Therefore, we believe that the different conclusions may be caused by different surgical experience and habits. In this study, all patients underwent surgery by the same team of spine surgeons, all of whom were senior associate chief physicians.

In this study, no significant difference was detected in functional and symptom outcome between the two groups at final follow-up. Compared with the pre-operative values, the post-operative VAS and ODI values were significantly improved in both groups. However, the percutaneous group showed significantly lower pain score than the paraspinal group at 3 days after surgery, which indicated that the percutaneous approach resulted in better short-term pain improvement. The percutaneous approach was superior to the paraspinal approach in terms of the lengths of incisions, intraoperative blood loss and duration of postoperative hospitalization. Early studies have shown that the distance of superficial points of the intermuscular plane and the midline decrease from S1 to L1 [28]. Actually the intermuscular space appears curvilinear in the axial plane, whose concavity facing the spinal elements and convexity facing the lateral spine. From S1 to L1 the intermuscular space is to rotate towards the spinous process plane around the outer edge of the facet joint, and then at the level of L1 the superficial point is close to the spinous process [29]. After blunt separation of the longissimus and multifidus muscles via Wiltse paraspinal approach, deep retractors are needed to pull the two muscles apart to clearly expose the operative field. Lateral traction is relatively easy, while medial traction can cause great soft tissue tension due to spinous process obstruction, which actually do much damage to neurovascular structure of soft tissue lateral and medial to the facet joint line respectively. At the same time, the authors also found that some muscle Spaces could not be identified on MRI, which meant that the muscle spaces of some patients could not be accurately identified during surgery [29]. Actually sometimes it is difficult to find the intermuscular plane accurately during operation and the blood vessels passing through the muscle are damaged, resulting in increased bleeding. In the anatomy report of thoracolumbar junction, Wang et al. pointed out that after the lumbar and dorsal fascia is cut open, the intermuscular space can't be found immediately [30]. Instead, we first see is the aponeurosis of erector spinae covering it, which is made up of the tendon of the thoracic longissimus muscle. In order to expose the intermuscular space, part of the longissimus tendon is often severed. Moreover, the Wiltse paraspinal approach procedure may cause some damage to the intertransverse muscle, the cephalic part of the facet joints, the posterior medial ramus of the spinal nerve that innervates the paravertebral soft tissue and the related ligaments. A human cadaver research by Regev et al. [31] indicated that the percutaneous approach was superior to the Wiltse paraspinal approach in terms of preserving the integrity of the multifidus innervation at the adjacent cranial level, when inserting pedicle screws. The percutaneous approach can clearly expose the entry point after the insertion of the working sleeve, freeing the assistant's hands. When the minimally invasive percutaneous sleeves are inserted through the puncture hole and the diameters increase gradually, it can compress the bleeding point in the muscle and stop the bleeding. The blood loss is less than the former approach. Therefore, the percutaneous approach conforms more to the minimally 
invasive concept that has been popular, which brings about faster recovery as a consequence of less trauma.

The current study also showed that the operation time of the percutaneous group was longer than that of the paraspinal group, but there was no no statistical difference between the two groups $(P>0.05)$. The lack of anatomic markers results in more operative procedure and more intraoperative $\mathrm{C}$-arm monitoring, which requires surgical physicians with enough anatomical imaging knowledge basis and experience in minimally invasive operation, especially in early cases. We also found that the fluoroscopy numbers were $18.52 \pm 3.54$ in percutaneous group and $6.31 \pm 2.18$ in paraspinal group $(P<0.05)$. While there is no consensus on the risks of long-term low-dose $\mathrm{X}$-ray exposure, the link between radiation and skin cancer and cataracts has long been a concern for surgeons. In addition, percutaneous approach is associated with more instruments and more complicated procedures, which leads to prolonged operation time to some extent. In the present study, we also compared the operative and post-operative costs of the percutaneous group ( $32.34 \pm 0.92$ thousand yuan) and the paraspinal group ( $28.63 \pm 0.64$ thousand yuan), with a significant difference $(p<0.05)$. Since different post-operative hospitalization time and similar treatment prescriptions between the two groups, the main reason for the cost difference was due to expensive hollow screw used in the percutaneous group. With the accumulation of surgical experience and the development of percutaneous procedure equipment, especially the gradual application of navigation, virtual reality and other technologies in surgery, the time of percutaneous pedicle screw surgery will be further shortened, the number of fluoroscopy will be significantly reduced, and intraoperative blood loss will be reduced accordingly, implant will be cheaper with better biomechanical performance and its advantages will be further highlighted.

In terms of safety, Chapman et al. [32] reported that the mal-placement rates of percutaneous group were similar to that of the open group after measurement for postoperative CT imaging for a series of 1609 screws by Gertzbein-Rao grading system. Similarly, some scholars argued that no statistically significant differences about the accuracy existed between the traditional open insertion and percutaneous placement according to Zdichavsky's scoring system [33]. Even Raley et al. [8] suggested that fluoroscopic guided percutaneous pedicle screw insertion in the thoracolumbar spine is associated with a low misplacement rate and an extremely low rate of complications compared with the high rates published in the literature for open method. Liu et al. [25] also discovered that no statistically difference existed between the Wiltse paraspinal and conventional open groups with no fusion in the accuracy rate of screw placement. We found no significant difference in accuracy of pedicle screw placement between the two groups, which is consistent with that published in literature [26].

In our study, two cases of fat liquefaction exudation suffered delayed incision healing in percutaneous group. One case of superficial surgical site infection who had a long history of type 2 diabetes, and two cases of skin edge necrosis were found in paraspinal group. Operation can raise the blood glucose levels of patients, which exerts influence on wound healing of diabetic cases. Guzman et al. [34] analyzed 2,568,994 degenerative lumbar spine procedures and stressed that diabetic patients had a higher risk of complications, including surgical site infection, than nondiabetic ones after lumbar spine surgery. The 
paraspinal approach requires a median incision similar to the traditional open method, and subcutaneous tissues separated, resulting in more trauma and bleeding, which increased the incidence of microorganisms entering the both superficial and deep wound site.

For the one case of delayed incision healing in the percutaneous group, we thought that the cause of fat liquefaction exudation might have been that the skin incision was not made lateral enough to the pedicle projection so that no appropriate angulation of the puncture needle can be made when inserting into the pedicle. More adjustment was required, pressing surrounding fat tissue. Raley et al. [8] clearly pointed out that a certain distance should be kept lateral to the outer edge of the pedicle projection during incision selection, so as to ensure that the puncture needle enters from the outer edge of the pedicle projection, and the adjustment of puncture direction can be reduced when passing through the pedicle. Meanwhile, in the paraspinal group, the surgeon sometimes deliberately reduces the length of the incision to pursue minimal invasive outcome, but in order to expose the surgical field sufficiently, he has to pull the skin forcefully during the operation, causing skin edge necrosis.

The guide wire breakage was thought as a result of the screw deviating from the trajectory of guide wire, which was transected when the screw was inserted. What's more, repeated use of the guide wire could also be one of the reasons. The anterior wall of the vertebra was broken because the guide wire was advanced by the tap unexpectedly. The great vessels were not injured by guide wire, which should be attributed to the fall as a result of the effect of gravity. This wire acts as a guide for all subsequent instruments, so it is imperative to keep the guide wire in place without inadvertently withdrawing or advancing it. To avoid this complication, prono-supination is required to withdrawn the needle. Keep the trajectory of guide wire by two needle holders such that it is not transected or bent by the tap or screws. The guide wire should be removed as soon as the screw tip exceeds the posterior vertebral wall.

\section{Conclusion}

In the treatment of thoracolumbar fractures without neurological defect, pedicle screw fixations via Wiltse paraspinal and percutaneous approach both can obtained minimally invasive and reliable effect, but the percutaneous pedicle screw fixation brought smaller trauma, less blood loss, longer operation time, more fluroscopy, higher surgery and postoperative costs, with their own unique complications especially in early learning curve. Despite that, it is still reasonable to believe that with the accumulation of surgical experience and the development of percutaneous procedure equipment, those shortcomings would be overcome, its advantages would be further highlighted, and the application range of this approach will become much broader.

\section{Abbreviations}

Visual Analog Scale $=$ VAS; Oswestry disability index $=$ ODI .

\section{Declarations}




\section{Funding}

No funding.

\section{Conflict of interest statement}

The authors declare that they have no conflicts of interest.

\section{Availability of data and material}

Some of the data involves patient privacy, and they can be obtained by contacting the corresponding author upon request.

\section{Code availability}

No code.

\section{Authors' contributions}

Chenghao Yu: Collect and analyze data, and write the article.

Fan Ding: Lead the research and revise the article.

Xiaosong Wu: Make suggestions for revising the article.

Zhengyun Ye: Make suggestions for revising the article.

Bing Hu: Make suggestions for revising the article.

\section{Compliance with ethical standards}

The Ethics Committee in The First People's Hospital of Jingmen approved the study.

\section{Consent to participate}

The co-authors declare that they have participated this research.

\section{Consent for publication}

The co-authors declare that they agree with the publication of this research.

\section{References}

1. GBD 2016 Neurology Collaborators (2019). Global, regional, and national burden of neurological disorders, 1990-2016: a systematic analysis for the Global Burden of Disease Study 2016. The Lancet. Neurology, 18(5), 459-480. 
2. Zhang C, Liu Y (2018). Combined pedicle screw fixation at the fracture vertebrae versus conventional method for thoracolumbar fractures: A meta-analysis. Int J Sur (London, England), 53, 38-47.

3. Liu B, Zhu Y, Liu S et al (2018). National incidence of traumatic spinal fractures in China: Data from China National Fracture Study. Medicine, 97(35), e12190.

4. Hitchon PW, Abode-lyamah K, Dahdaleh NS et al (2016). Nonoperative Management in Neurologically Intact Thoracolumbar Burst Fractures: Clinical and Radiographic Outcomes. Spine, 41(6), 483-489.

5. Pehlivanoglu T, Akgul T, Bayram S et al (2020). Conservative Versus Operative Treatment of Stable Thoracolumbar Burst Fractures in Neurologically Intact Patients: Is There Any Difference Regarding the Clinical and Radiographic Outcomes?. Spine, 45(7), 452-458.

6. Styf JR, Willen J (1998). The effects of external compression by three different retractors on pressure in the erector spine muscles during and after posterior lumbar spine surgery in humans. Spine, 23(3), 354-358.

7. Ghiasi MS, Arjmand N, Shirazi-Adl A et al (2016). Cross-sectional area of human trunk paraspinal muscles before and after posterior lumbar surgery using magnetic resonance imaging. Eur Spine $\mathrm{J}$, 25(3), 774-782.

8. Raley DA, Mobbs RJ (2012). Retrospective computed tomography scan analysis of percutaneously inserted pedicle screws for posterior transpedicular stabilization of the thoracic and lumbar spine: accuracy and complication rates. Spine, 37(12), 1092-1100.

9. Wiltse LL, Bateman JG, Hutchinson RH et al (1968). The paraspinal sacrospinalis-splitting approach to the lumbar spine. J Bone Joint Surg Am, 50(5), 919-926.

10. Wiltse LL, Spencer CW (1988). New uses and refinements of the paraspinal approach to the lumbar spine. Spine, 13(6), 696-706.

11. Liu JH, Pang ZB, Xu WB et al (2017). Comparison of pedicle fixation by the Wiltse approach and the conventional posterior open approach for thoracolumbar fractures, using MRI, histological and electrophysiological analyses of the multifidus muscle. Eur Spine J, 26(5):1506-1514.

12. Chang W, Zhang D, Liu W et al (2018). Posterior paraspinal muscle versus post-middle approach for the treatment of thoracolumbar burst fractures: A randomized controlled trial. Medicine (Baltimore), 97(25): e11193.

13. Chen ZD, Wu J, Yao XT et al (2018). Comparison of Wiltse's paraspinal approach and open book laminectomy for thoracolumbar burst fractures with greenstick lamina fractures: a randomized controlled trial. J Orthop Surg Res,13(1):43.

14. Mathews HH, Long BH (1995)区Endoscopy assisted percutaneous anterior interbody fusion with subcutaneous suprafascial internal fixation: evolution of technique and surgical considerations. Orthop Int, 3: 496-500.

15. Foley KT, Gupta SK (2002). Percutaneous pedicle screw fixation of the lumbar spine: preliminary clinical results. J Neurosurg, 97(1 Suppl):7-12. 
16. Kim DY, Lee SH, Chung SK et al (2005). Comparison of multifidus muscle atrophy and trunk extension muscle strength: percutaneous versus open pedicle screw fixation. Spine, 30(1):123-9.

17. Yang M, Zhao Q, Hao D et al (2019). Comparison of clinical results between novel percutaneous pedicle screw and traditional open pedicle screw fixation for thoracolumbar fractures without neurological deficit. Int Orthop, 43(7): 1749-1754.

18. Gong Y, Fu G, Li B et al (2017). Comparison of the effects of minimally invasive percutaneous pedicle screws osteosynthesis and open surgery on repairing the pain, inflammation and recovery of thoracolumbar vertebra fracture. Exp Ther Med,14(5): 4091-4096.

19. Kocis J, Kelbl M, Kocis T et al(2020). Percutaneous versus open pedicle screw fixation for treatment of type A thoracolumbar fractures. Eur J Trauma Emerg Surg, 46(1):147-152.

20. Sun X Y, Zhang X N, Hai Y (2017). Percutaneous versus traditional and paraspinal posterior open approaches for treatment of thoracolumbar fractures without neurologic deficit: A meta-analysis. Eur Spine J, 26(5):1418-1431.

21. Sanderson PL, Fraser RD, Hall DJ (1999). Short segment fixation of thoracolumbar burst fractures without fusion. Eur Spine J, 8(6):495-500.

22. Jindal N, Sankhala SS, Bachhal V(2012). The role of fusion in the management of burst fractures of the thoracolumbar spine treated by short segment pedicle screw fixation: a prospective randomised trial. J Bone Joint Surg Br, 94(8):1101-1106.

23. Diniz JM, Botelho RV (2017). Is fusion necessary for thoracolumbar burst fracture treated with spinal fixation? A systematic review and meta-analysis. J Neurosurg Spine, 27(5):584-592.

24. Lee JK, Jang JW, Kim TW et al (2013). Percutaneous short-segment pedicle screw placement without fusion in the treatment of thoracolumbar burst fractures: is it effective?: comparative study with open short-segment pedicle screw fixation with posterolateral fusion. Acta Neurochir (Wien), 155(12):2305-2312.

25. Liu Z, Li Z, Xing D et al (2015). Two different surgery approaches for treatment of thoracolumbar fracture. Int J Clin Exp Med, 8(12):22425-22429.

26. Fan Y, Zhang J, He X et al (2017). A Comparison of the Mini-Open Wiltse Approach with Pedicle Screw Fixation and the Percutaneous Pedicle Screw Fixation for Neurologically Intact Thoracolumbar Fractures. Med Sci Monit, 23(1):5515-5521.

27. Fitschen-Oestern S, Scheuerlein F, Weuster M et al (2015). Reduction and retention of thoracolumbar fractures by minimally invasive stabilisation versus open posterior instrumentation. Injury, 46(1): S63-S70.

28. Palmer DK, Allen JL, Williams PA et al (2011). Multilevel magnetic resonance imaging analysis of multifidus-longissimus cleavage planes in the lumbar spine and potential clinical applications to Wiltse's paraspinal approach. Spine, 36(16):1263-1267.

29. Li H, Yang L, Chen J et al (2016). Magnetic resonance imaging-based anatomical study of the multifidus-longissimus cleavage planes in the lumbar spine. Am J Transl Res, 8(1):109-116. 
30. Wang JY, Deng XF, Liao WB et al (2015). Anatomic and clinical study of paraspinal intermuscular space in the thoracolumbar spine. Acta Universitatis Medicinalis Anhui, 50(2):164-168.

31. Regev GJ, Lee YP, Taylor WR et al (2009). Nerve injury to the posterior rami medial branch during the insertion of pedicle screws: comparison of mini-open versus percutaneous pedicle screw insertion techniques. Spine, 34(11):1239-1242.

32. Chapman TM, Blizzard DJ, Brown CR (2016). CT accuracy of percutaneous versus open pedicle screw techniques: a series of 1609 screws. Eur Spine J, 25(6):1781-1786.

33. Chiu CK, Chan CYW, Kwan MK (2017). The accuracy and safety of fluoroscopic-guided percutaneous pedicle screws in the thoracic and lumbosacral spine in the Asian population: A CT scan analysis of 1002 screws. J Orthop Surg, 25(2): 2309499017713938.

34. Guzman JZ, latridis JC, Skovrlj B et al (2014). Outcomes and complications of diabetes mellitus on patients undergoing degenerative lumbar spine surgery. Spine, 39(19):1596-1604.

\section{Tables}


Table 1

Comparison of general data between two groups

\begin{tabular}{|c|c|c|c|c|}
\hline & percutaneous group & paraspinal group & Statistical value & $P$ value \\
\hline Cases & 48 & 50 & & \\
\hline Sex(Male/Female) & $26 / 22$ & $29 / 21$ & $\chi^{2}=0.146$ & $>0.05$ \\
\hline Age(year) & $40.98 \pm 10.80$ & $42.74 \pm 9.76$ & $t=-0.848$ & $>0.05$ \\
\hline BMI & $21.34 \pm 2.13$ & $21.20 \pm 1.96$ & $t=0.323$ & $>0.05$ \\
\hline Fracture segment(n) & & & $\chi^{2}=0.380$ & $>0.05$ \\
\hline T11 & 9 & 11 & & \\
\hline T12 & 14 & 16 & & \\
\hline L1 & 15 & 14 & & \\
\hline L2 & 10 & 9 & & \\
\hline AO fracture classification & & & $\chi^{2}=0.403$ & $>0.05$ \\
\hline A2 & 17 & 15 & & \\
\hline A3 & 14 & 17 & & \\
\hline B1 & 17 & 18 & & \\
\hline Injury cause(n) & & & $\chi^{2}=0.787$ & $>0.05$ \\
\hline Fall down & 18 & 17 & & \\
\hline Traffic accident & 14 & 18 & & \\
\hline Trip & 10 & 8 & & \\
\hline Crushed by heavy object & 6 & 7 & & \\
\hline
\end{tabular}


Table 2

Comparison of perioperative indexes $(\overline{\mathrm{x}} \pm \mathrm{s}$ ) between two groups

\begin{tabular}{|c|c|c|c|c|}
\hline & $\begin{array}{l}\text { percutaneous } \\
\text { group }\end{array}$ & $\begin{array}{l}\text { paraspinal } \\
\text { group }\end{array}$ & t value & $\begin{array}{l}\mathrm{P} \\
\text { value }\end{array}$ \\
\hline Incision length(cm) & $7.25 \pm 0.55$ & $10.64 \pm 0.56$ & -7.545 & $<0.05$ \\
\hline Operative time(min) & $75.47 \pm 5.42$ & $72.12 \pm 5.29$ & 1.525 & $>0.05$ \\
\hline Intraoperative blood loss(ml) & $62.26 \pm 8.94$ & $74.64 \pm 8.61$ & -3.818 & $<0.05$ \\
\hline Fluoroscopy number & $18.52 \pm 3.54$ & $6.31 \pm 2.18$ & 4.472 & $<0.05$ \\
\hline Postoperative hospital stay(d) & $5.81 \pm 1.12$ & $8.00 \pm 1.26$ & -10.330 & $<0.05$ \\
\hline $\begin{array}{l}\text { Operative and post-operative costs } \\
\text { (thousand yuan) }\end{array}$ & $32.34 \pm 0.92$ & $28.63 \pm 0.64$ & 5.443 & $<0.05$ \\
\hline
\end{tabular}

Table 3

Comparison of imaging parameters ( $\overline{\mathrm{x}} \pm \mathrm{s}$ ) between two groups

\begin{tabular}{|c|c|c|c|c|c|}
\hline & time point & $\begin{array}{l}\text { percutaneous } \\
\text { group }\end{array}$ & $\begin{array}{l}\text { paraspinal } \\
\text { group }\end{array}$ & $\begin{array}{l}\mathrm{t} \\
\text { value }\end{array}$ & $\begin{array}{l}\mathrm{P} \\
\text { value }\end{array}$ \\
\hline \multirow{3}{*}{$\begin{array}{l}\text { percentage of the anterior } \\
\text { vertebral height }(\%)\end{array}$} & preoperative & $64.92 \pm 9.07$ & $63.70 \pm 9.19$ & 0.66 & $>0.05$ \\
\hline & $\begin{array}{l}1 \text { week } \\
\text { postoperative }\end{array}$ & $93.61 \pm 1.37 *$ & $93.26 \pm 1.24 *$ & 1.322 & $>0.05$ \\
\hline & $\begin{array}{l}1 \text { year } \\
\text { postoperative }\end{array}$ & $90.12 \pm 1.05^{\star \#}$ & $89.78 \pm 1.52^{\star \#}$ & 1.264 & $>0.05$ \\
\hline \multirow[t]{3}{*}{ Cobb angle of kyphosis $\left({ }^{\circ}\right)$} & preoperative & $17.58 \pm 2.58$ & $17.42 \pm 2.56$ & 0.314 & $>0.05$ \\
\hline & $\begin{array}{l}1 \text { week } \\
\text { postoperative }\end{array}$ & $6.44 \pm 1.13^{*}$ & $6.28 \pm 0.99 *$ & 0.735 & $>0.05$ \\
\hline & $\begin{array}{l}1 \text { year } \\
\text { postoperative }\end{array}$ & $9.73 \pm 1.16^{\star \#}$ & $10.08 \pm 1.47^{\star \#}$ & -1.308 & $>0.05$ \\
\hline
\end{tabular}


Table 4

Comparison of functional and symptom results $(\overline{\mathrm{x}} \pm \mathrm{s})$ between two groups

\begin{tabular}{|c|c|c|c|c|c|}
\hline & time point & percutaneous group & paraspinal group & $\mathrm{t}$ value & $P$ value \\
\hline \multirow[t]{4}{*}{ VAS } & preoperative & $7.94 \pm 0.84$ & $8.06 \pm 0.84$ & -0.722 & $>0.05$ \\
\hline & 3 days postoperative & $3.66 \pm 0.93^{\star}$ & $4.76 \pm 0.62^{\star}$ & -6.856 & $<0.05$ \\
\hline & 6 months postoperative & $1.81 \pm 0.57 * \#$ & $1.88 \pm 0.68^{\star \#}$ & -1.586 & $>0.05$ \\
\hline & 1 year postoperative & $1.02 \pm 0.67^{\star \# \Delta}$ & $1.16 \pm 0.68^{\star \# \Delta}$ & -1.021 & $>0.05$ \\
\hline \multirow[t]{3}{*}{ ODI } & preoperative & $46.33 \pm 7.06$ & $47.40 \pm 5.58$ & -0.832 & $>0.05$ \\
\hline & 6 months postoperative & $14.50 \pm 2.49 *$ & $15.04 \pm 2.89 *$ & -1.125 & $>0.05$ \\
\hline & 1 year postoperative & $5.46 \pm 1.88^{\star \Delta}$ & $5.32 \pm 1.60 * \Delta$ & 0.393 & $>0.05$ \\
\hline
\end{tabular}

Table 5

Comparison of accuracy grading of pedicle screws between two groups

\begin{tabular}{|lcccll|}
\hline & Grade 0 & Grade 1 & Grade 2 & $\chi^{2}$ & P value \\
\hline percutaneous group & 185 & 4 & 3 & 0.446 & 0.8 \\
\hline paraspinal group & 190 & 6 & 4 & & \\
\hline
\end{tabular}

